\title{
Serotonin (5-HT) Increases Olfactory Responsiveness in the Tobacco Hawkmoth Manduca sexta
}

\author{
Samantha Lynn Jusino \\ West Virginia University
}

Follow this and additional works at: https://researchrepository.wvu.edu/etd

\section{Recommended Citation}

Jusino, Samantha Lynn, "Serotonin (5-HT) Increases Olfactory Responsiveness in the Tobacco Hawkmoth Manduca sexta" (2014). Graduate Theses, Dissertations, and Problem Reports. 407.

https://researchrepository.wvu.edu/etd/407

This Thesis is protected by copyright and/or related rights. It has been brought to you by the The Research Repository @ WVU with permission from the rights-holder(s). You are free to use this Thesis in any way that is permitted by the copyright and related rights legislation that applies to your use. For other uses you must obtain permission from the rights-holder(s) directly, unless additional rights are indicated by a Creative Commons license in the record and/ or on the work itself. This Thesis has been accepted for inclusion in WVU Graduate Theses, Dissertations, and Problem Reports collection by an authorized administrator of The Research Repository @ WVU. For more information, please contact researchrepository@mail.wvu.edu. 
Serotonin (5-HT) Increases Olfactory Responsiveness in the Tobacco Hawkmoth Manduca sexta

Samantha Lynn Jusino

Thesis submitted

to the Eberly College of Arts and Sciences

at West Virginia University

in partial fulfillment of the requirements for the degree of

Master of Science in

Biology

Kevin C. Daly, Ph.D., Chair

Andrew Dacks, Ph.D.

Jorge Flores, Ph.D.

Department of Biology

Morgantown, West Virginia

2014

Keywords: Serotonin, Manduca, detection, generalization, olfaction neuromodulation

Copyright 2014 Samantha Lynn Jusino 


\title{
ABSTRACT \\ Serotonin (5-HT) Increases Olfactory Responsiveness in the Tobacco Hawkmoth Manduca sexta
}

\author{
Samantha Jusino
}

Behavioral states of animals change throughout the day and the brain has developed several mechanisms in order for neural circuits to modify network function in order to produce these states. Neuromodulation allows the animal to alter neural circuits to make them more or less responsive to a given input. Little research has been conducted to understand the functional role of serotonin (5-HT), a neuro modulator, on olfactory behavioral responses. In this study, we aimed to identify the functional role of 5-HT in Manduca sexta olfactory function. Using a Pavlovian-Conditioning Paradigm, we conditioned moths to 2-hexanone and tested them using two different assays, a detection threshold assay and a generalization gradient assay. We found that 5-HT significantly increased olfactory sensitivity and significantly sharpened generalization gradients. Blocking 5-HT had no effect on detection thresholds but did lower behavioral responsiveness to odors in the generalization gradient assay. These results support similar studies focused on pheromone processing and perception and indicate that 5HT tunes the sensitivity of the olfactory system. 


\section{Acknowledgements}

I would like to thank all of the people who contributed to the successful completion of this thesis. I would like to start by thanking my committee, Dr. Kevin C. Daly, Dr. Andrew M. Dacks, and Dr. Jorge Flores. My advisor for this project, Kevin C. Daly, has taught me a large amount about insect neuroethology and without his guidance and support throughout the course of this project, I would have not made it. He has pushed me to be a better student, researcher, and teacher and ultimately helped me find my true passion and for that I am grateful. Drs. Dacks and Flores have provided tremendous support serving on my committee and helping me design my overall experiment.

Next, I would like to thank all of my lab members who have helped over the past 4 years, Rex Burkland, Sam Bradley, and especially Phil Chapman. Rex taught me all the methods that I used for this thesis. Sam has been a tremendous help with looking over drafts and a tremendous help with analyzing my data. Phil was an integral part of finishing the data collection. He was my blind tester throughout the duration of my thesis research, without his help, I would not have been able to succeed.

Lastly, I would like to thank my family and friends. They have provided a support system like no other. They have always been the force guiding me to succeed and to them I am eternally grateful. 


\section{Table of Contents}

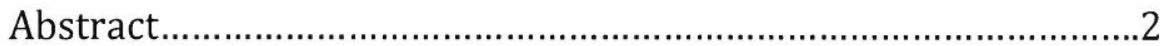

Acknowledgements..................................................................

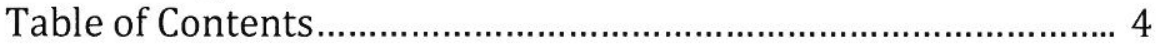

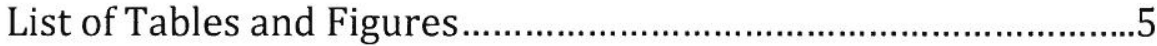

I. Chapter I: Literature Review.......................................................6-13

II. Chapter II: Methodological Approaches........................................14-25

III. Chapter III: Results................................................................26-33

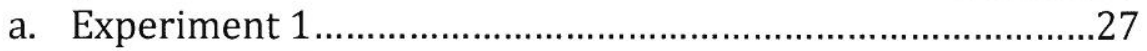

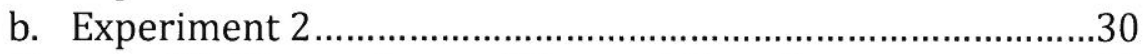

IV. Chapter IV: Discussion and Future Directions......................31-42

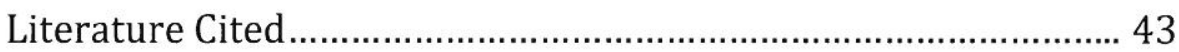




\section{List of Tables and Figures}

Figure 1 Experimental Setup

Figure 2 -

Injection site of Manduca

Figure 3

-page 17

Experimental protocol and design for Experiment 1: Detection Threshold

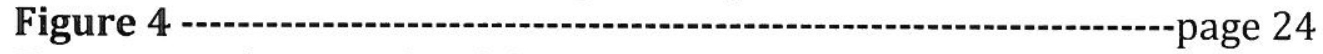

Experimental protocol and design for Experiment 2: CS Generalization Gradient

Figure 5

-page 29

Results of Experiment 1: 5-HT Significantly Increases Olfactory Sensitivity Figure 6

Tukey's Grouping of Odors Concentrations

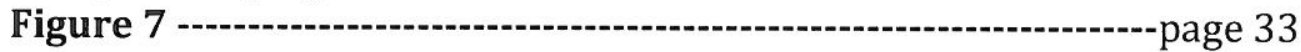

Results of Experiment 2: 5-HT Sharpens the Generalization Gradient 
Chapter 1: Introduction 


\section{Introduction:}

Animal nervous systems consist of complex networks of cells that have developed specific functions. These networks are able to communicate between each other, and have evolved over time to become flexible and change with the needs of the animal. Inputs to these networks are variable and the nervous system has developed neuromodulatory mechanisms to alter the efficacy of neural circuits in order to cope with the changes. Neuromodulators can alter individual neurons thereby affecting the networks in which they are apart of, changing the circuit in multiple ways. Thus, neuromodulators add a level of tuning to neural networks/circuits.

Neuromodulation is the process by which neuromodulatory substances alter the relationship between the synapses by changing the amount of neurotransmitter that is released, regardless of the receptor type that it acts upon. This can be achieved by changing both the firing and physiological properties of the neuron. Serotonin (5-HT) is a neuromodulatory substance in both vertebrates and insects and has been extensively studied, particularly in the Manduca sexta (tobacco hawmoth) olfactory system. To date, the behavioral consequence of 5-HT has yet to be characterized in Manduca. By exploring the effects of 5-HT application in the antennal lobe $(\mathrm{AL})$ we will be able to provide a functional role for 5-HT in olfactory behavior to non-pheromonal odors.

In the insect olfactory system, odors are first detected by olfactory receptor neurons (ORNs), which are housed in the antenna within the sensilla (Lee et al. 1990). ORNs project up into the AL of insects where they synapse in glomeruli. 
Glomeruli are small spherical structures of synaptic neuropil. There are approximately 63 different glomeruli in both males and females (Rospars \& Hildebrand 1992). In addition to housing the axonal projections of ORNs, glomeruli also contain terminals of local interneurons (LNs) and projection neurons (PNs) which exit the AL and ramify the protocerebrum.

Serotonergic centrifugal neurons are also present in the AL and send processes to all of the glomeruli (Kent et al. 1987). Manduca have a bilateral pair of contralaterally projecting serotonergic immunoreactive neurons, each of which project to and ramify the contralateral AL (Kent et al. 1987). These neurons were termed the contralaterally projecting 5-HTir duetocerebral neuron (CSD neuron; Dacks et al. 2006a) because of their projection patterns. These neurons project in the dorsoposterior direction to the protocerebrum and cross the midline posteriorally at the central body then project into the contralateral AL where they arborize in all glomeruli (Kent et al. 1987). The morphology of these neurons suggests that Manduca may be using 5-HT to modulate the sensory input into the AL. however they not likely to directly affect ORNs due to the lack of direct overlap of the CSD neuron with the sensory input in immuno labeling studies (Sun et al. 1993).

However, electrophysiological studies indicate that $5 \mathrm{HT}$ alters channel conductances in PNs and therefore may provide evidence about how 5-HT modulates olfactory circuits. For example, potassium channels $\left(\mathrm{K}^{+}\right)$are responsible for the repolarization of the neuron back to its resting membrane potential. In Manduca, two different $\mathrm{K}^{+}$channels affected by $5-\mathrm{HT}$ are $\mathrm{I}_{\mathrm{A}}$ and $\mathrm{I}_{\mathrm{K}(\mathrm{V})}$. $\mathrm{I}_{\mathrm{A}}$ is a fast acting 
transient current responsible for hyperpolarization phase of the action potential whereas $I_{k(v)}$ is a slower acting delayed rectifier current responsible for the refractory period. In Manduca AL, 5-HT application appears to decreases voltage gated $\mathrm{I}_{\mathrm{A}} \mathrm{K}^{+}$current conductance in PNs (Kloppenburg et al. 1999). Furthermore, $\mathrm{I}_{\mathrm{A}}$ channels were affected by 5-HT via two different mechanisms; a decrease in conductance, and a shifted half-max inactivation voltage down by approximately $7 \mathrm{mV}$ (Kloppenburg et al. 1999). This indicates that $5-\mathrm{HT}$ is not only reducing the conductance of the channel during the activation of the channel but also makes the channel more likely to become inactivated. Reducing the conductance of the $\mathrm{K}^{+}$ channel can lead to an overall increase in the excitability of the PN as well as extend the overall duration of an action potential, which can increase transmitter release.

For example, experiments using electrically evoked PN responses indicate that 5-HT induces spike broadening by changing the efficacy of the $\mathrm{K}^{+}$channels. Spike broadening in turn increases the duration of the inward $\mathrm{Ca}^{2+}$ current within axon terminals hence altering the excitability of the cell (Kloppenburg \& Mercer 2008). Thus, it is plausible to suggest that higher cell excitability would lead to a prolonged action potential. This can be observed when looking at cell resistance. 5HT causes an increase in the cell input resistance of PNs in addition to increasing overall cell excitability (Kloppenburg \& Hildebrand 1995). This is not surprising as reducing a fast acting transient current should prolong the return of voltage to the resting potential.

Spike broadening is one means to indirectly affect associative strength of sensory stimuli. By increasing the input resistance and allowing the cell to be active 
longer for each action potential. This change in sensory response would lead to an increase in stimulus salience allowing the animal to predict the onset of unconditioned stimulus (US) from the conditioned stimuli (CS) more efficiently (Rescorla \& Wagner 1972). By increasing stimulus salience it would allow the animal to be more responsive to a particular stimulus. This would be consistent with the idea of neuromodulation making a system more sensitive to behaviorally relevant stimuli.

The previously described effects of 5-HT on channel conductances can be good indicators of how 5-HT will act on odor-evoked response. 5-HT has been investigated with regards to how pheromones elicit responses of PNs. In one study, an increase in the magnitude and duration of the evoked potentials during pheromone stimulation to the antenna when 5-HT was applied (Kloppenburg \& Heinbockel 2000). These results suggest that there is an increase in the activity of pheromone-sensitive neurons isolated in the macroglomerular complex (MGC) of the AL. The MGC is a 3 glomerular structure that is sexually dimorphic to the male (Christensen \& Hildebrand 1987; Hildebrand \& Shepherd 1997). The CSD neuron not only ramifies the MGC but all glomeruli and therefore can affect non-pheromone odor responses.

5-HT has been shown to modulate non-pheromone odors. In Manduca, 5-HT increased the response of a group of neurons as well as an overall enhancement of the response at the highest concentration of non-pheromone odors (Dacks et al. 2008). Based on this study, 5-HT may be increasing the resolution for AL coding odor intensity thus increasing the responsiveness to odors over a given range of 
concentrations (Dacks et al. 2008). Ideally, when 5-HT is applied, the cells affected will be more likely to respond to odors across a given concentration range making the system more sensitive. Furthermore, in Drosophila, 5-HT application was shown to lower the response threshold of PNs in the presence of non-pheromone odors (Dacks et al. 2009). Therefore, it may be predicted from the odor-evoked potential, that when 5-HT is added to the AL in Manduca, it should be observed that the animal would be able to detect odor concentrations below the normal detection threshold making the animal more sensitive to the stimulus.

The levels of 5-HT in the Manduca fluctuate throughout the light/dark cycle. Analysis of 5-HT concentration in the AL showed that the level of 5-HT was different when measured throughout the day (Kloppenburg et al. 1999). Manduca are crepuscular animals meaning that they are active at dusk and dawn. In a 24-hour period, there were levels of 5-HT increased at the onset of duck and peaked during the dark cycle of the day and lower amounts of 5-HT during the light cycle or relative day of the animal. This pattern was also observed with the onset of the relative day. The ecology of the animal suggests that $5-\mathrm{HT}$ would be modulating the olfactory system during the active period of the day when the animal would be foraging, performing mate seeking behaviors and reproducing. Modulating the system would make it more sensitive during this period. The pattern in which the amount of 5-HT fluctuates throughout the day follows a circadian like pattern and suggesting that 5-HT could play a role in sensory arousal/attention.

To date, there have only been a few studies that examine the behavioral consequences of 5-HT on insects. In Bombyx mori, 5-HT increased the percentage of 
behavioral response to pheromone (Gatellier et al. 2004). Four animals were placed in a box and exposed to varying concentrations of Bombykal, the pheromone of $B$. mori. The behavioral response to the odor was wing fanning within the first 30secs of the pheromone stimulus. The modulatory effects of 5-HT allowed the animal to detect odors at a lower concentration of Bombykal (Gatellier et al. 2004). These results are consistent with the results of the electrically evoked responses and odorevoked responses; however, these results are limited to behavioral consequences of 5-HT application in response to pheromones.

In the current experiment, we examined the behavioral consequences of 5HT modulation in the olfactory system of Manduca. 5-HT has been shown to have an overall enhancement of sensitivity of the olfactory system in Manduca by altering the conductance of two $\mathrm{K}^{+}$channels. Changing the properties of $\mathrm{I}_{\mathrm{A}}$ allows the neuron to be excited longer thus prolonging the action potential, indicating suggest spike broadening. This overall enhancement of the olfactory system is also observed when the animal is presented with odors as well. In both Manduca and Drosophila, 5-HT had an overall enhancement of odor detection to both pheromones and nonpheromone odors. Fluctuating levels of 5-HT in the AL suggests that higher levels of 5-HT is regulating state-dependent changes in the animal. The aim of this project was to characterize the behavioral consequence of 5-HT in the AL of Manduca. Using a classical Pavlovian conditioning paradigm, olfactory responsiveness was measured and used as a basis for detection threshold and CS generalization experiments. Based on prior experiments, the hypothesis was that application of 5HT will increase olfactory sensitivity and induce an increase in the feeding muscle 
activity of the animal; whereas methiothepin application, a 5-HT1 1 receptor antagonist with cause a decrease in the behavioral response. Overall, this study would implicate a role for 5-HT in behavioral sensitivity to odor processing. 
Chapter 2: Methodological Approach 


\section{Methods:}

Subjects:

Male and female Manduca sexta moths were reared in a colony at West Virginia University's Biology Department. Larvae were fed a wheatgerm-based diet and were placed in pupation chambers as they entered the final "wandering" stage of larval development. At about stage 18 of pupal development, individuals were classified based on sex and kept individually in brown paper bags which were placed in an incubator with an $18 \mathrm{~h}: 6 \mathrm{~h}$ reverse light dark schedule at $25^{\circ} \mathrm{C}$ with $75 \%$ relative humidity. Pupae were checked daily for newly emerged adults and eclosion dates were marked. Subjects used were 5-7 days post eclosion. Animals were food deprived post eclosion in order to increase motivation to feed on sucrose solution. They were randomly assigned to each condition.

\section{Preparation:}

Moths were inserted head first into a tube with an inner diameter of $2.54 \mathrm{~cm}$. Prior to insertion into the tube, scales from the moths were removed from the head capsule and thorax. The head came out and over a $0.75 \mathrm{~cm}^{2}$ raised tab at the top of the tube. The moth is then held snug to the tube using a piece of tape, keeping the moth's head immobilized against the tube, and allowed for a stable platform for dissection. A Teflon coated silver elctromyographic (EMG) electrode was implanted through the cuticle just above the right cibarial pump muscle that is involved in feeding behavior (Eaton 1971). A reference electrode of the same material was placed in the contralateral eye. Electrodes were super-glued in place to ensure that they did not come loose during the dissection. Impedance of the electrical circuit 
was tested using a FHC low voltage impedance meter to confirm quality of the circuit $(0.1 \mathrm{M} \Omega-0.8 \mathrm{M} \Omega$ was the acceptable range). The proboscis was then threaded through a $4 \mathrm{~mm}$ length Tygon tubing and affixed to the tube using soft dental wax. The tube with the prepared moth was then affixed on a mobile platform that connected the electrode leads to a plug on the conditioning and testing stage, which then lead to an amplifier.

\section{Stimulus Delivery:}

Figure $\mathbb{1}$ highlights some of the key aspects of the conditioning and testing apparatus. The stimulus delivery stage used for conditioning and testing was composed of an odor delivery system and an evacuation vent. The evacuation vent was used to ensure that that the odor from the previous trials was removed prior to the new trial. Naïve animals were placed in a constant airflow from the exhaust of approximately $0.3 \mathrm{~m} / \mathrm{s}$. An odor cartridge was secured in a base using dental wax and placed $10 \mathrm{~cm}$ upwind. The odor cartridge tip was aimed directly at the head and antenna. A programmable logic chip (PLC) was used to control both airflow and timing and delivery of the odor.

Air was supplied through a central airline. The air was filtered through a $500 \mathrm{cc}$ Drierite cartridge; this was used to extract moisture from the air and then air passed through a 500cc cartridge of active charcoal to extract any organic compounds. Following the filtration system, air passed into a 3-way Lee brand valve, which was controlled by the PLC. The air flowed into an open port and then immediately out a second valve. When the valve was activated, the air was forced into the third valve (normally closed) that was connected to the odor cartridge via 
Tygon brand tubing. The final airflow was calibrated using a flow meter to $250 \mathrm{~mL} / \mathrm{min}(\sim 90 \mathrm{~cm} / \mathrm{sec})$; this flow rate along with the cartridge nozzle and distance configuration ensured that the odor was dispersed into a plume of approximately $4 \mathrm{~cm}$ in diameter, wide enough to cover both antennae.

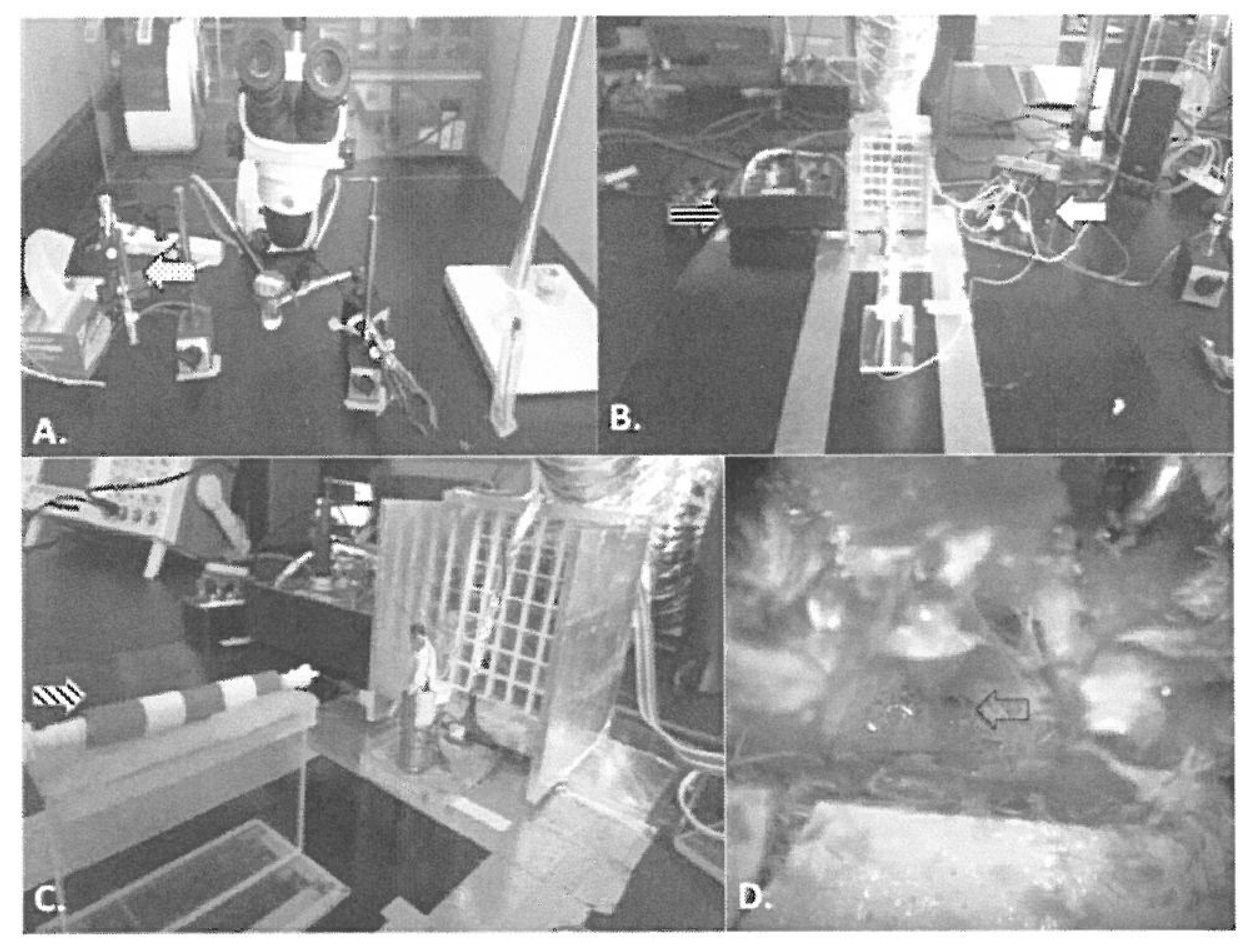

Figure 1. Experimental Setup. This figure displays the experimental setup used for each stage of the experiment. (A) Panel A shows the dissecting scope, the animal was secured in the clamp and then positioned under the scope in preparation for the placement of the electrode into the antennal lobe. The polka dotted arrow points to the micromanipulator that was connected to the picoinjector and housed the quartz glass electrode. The electrode was calibrated in a pool of mineral oil so that the droplet was no larger than 2nL. (B) Frontal view of the animal on the testing rig. The odor is released based on a computer program and controlled using a programmable logic chip (solid white arrow). The base plugs into a stage that is connected to an amplifier (horizontal line arrow) and oscilloscope. (C) A side view of the animal plugged into the testing stage. The air cartridge was position $10 \mathrm{~cm}$ away from the antenna (diagonal line arrow) (D). Injection site where the drug or vehicle was injected. The blue dye indicates where the injection is placed (open arrow). This shows that the injection is localized and not a widespread application as opposed to bath application of a drug. 
The odor cartridge was made of glass tubing with an inner diameter of $6 \mathrm{~mm}$. It was fitted using Cole Palmer nylon lure fittings. The final internal volume of the cartridge was $1.7 \mathrm{~mL}$ once the fittings were in place. A small piece of Whatmans \#1 filter paper was inserted into the glass cartridge upon which odor was placed.

\section{Conditioning Protocol:}

An associative forward-paired Pavlovian conditioning protocol was used to condition the moths. There were a total of 6 conditioning trials and a series of extinction trials (test trials) 24 hours post training. For training, each prepared moth was placed onto the stage and connected to the amplifier. Each moth received a $4 \mathrm{~s}$ pulse of air from the odor cartridge, containing $3 \mu \mathrm{L}$ of undiluted 2-hexanone, (CS) which was directed at the antennae; 3 s into CS presentation $5.0 \mu \mathrm{L}$ of $0.75 \mathrm{M}$ sucrose solution was applied to the exposed proboscis (US) for 4s. This resulted in a 1 second overlap of the CS and US. There was a 6-minute interval between conditioning trials. Three indicators of that response were used: a visual change in the firing pattern from the oscilloscope after presentation of the CS, audio output from the speakers connected to the amplifier, and an extension of the exposed proboscis in response to the CS. Evidence from any one or more of these indicators were used.

Dissection and Injection Protocols:

Approximately 15 minutes prior to testing, the caudal end of the head capsule of the conditioned moth was opened and the ALs (AL) were exposed. The existing musculature and proboscis did not have to be removed; however, the 
cibarial pump muscle was repositioned in order to gain better access to the AL. A rhombus shaped piece of cuticle was cut above the muscle and removed. The cut line was then extended down the antennal line to the base of the capsule and the cibarial pump was repositioned forward and secured to the top of the capsule using super glue (Figure 2). This procedure has been previously used and had no discernable effect on the ability to illicit a normal feeding behavior (Mwilaria et al. 2008).

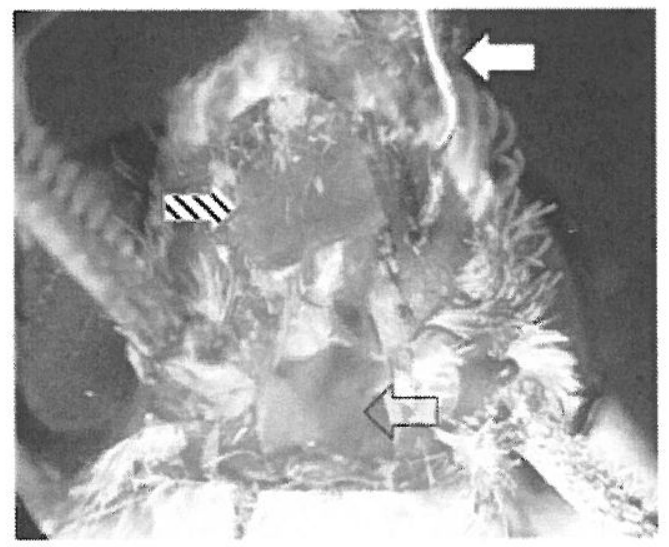

Figure 2. Injection site of Manduca. A small rhomboid shaped piece of the head cuticle was cut away and removed. The cut was then expanded down the antennal line and the ciribal pump muscle was positioned forward and secured using super glue (diagonal line arrow). Trachea was then cut away until the AL was exposed (black arrow). There were two wire electrodes, one placed just below the cuticle in contact with the muscle (white arrow) and the other placed in the eye as a reference electrode.

A sharp quartz intracellular electrode was pulled using a Sutter P2000 laser electrode puller to create a slow tapering injection probe. The probe tips were broken in order to create a $\sim 10 \mu \mathrm{m}$ diameter. The electrode was calibrated in a mineral oil pool before and after each injection to ensure that the volume of the droplet remained constant $(2 \mathrm{~nL})$. The probe was then positioned into the $\mathrm{AL}$ between the two large and identifiable tracheal branches wrapping the AL. The 
injection was delivered with a pressure of $20 \mathrm{psi}$ and the injection time was in the range of $18 \mathrm{msec}$; the range varied to calibrate the injection volume. If after the injection, the droplet volume was different in size or the electrode was clogged, the animal was discarded from the experiment. Post injection, a 15-minute wait period was used to allow the animals to "recover" from the effects of the dissection and injection. A 20-minute bath application of 5-HT was used in electrophysiological studies (Dacks et al. 2008). Because the injection is directly in the AL and does not need to diffuse into it, a slightly shorter wait period was used.

The physiological saline used as the drug vehicle was injected into control animals $\left(150 \mathrm{mM} \mathrm{NaCl}, 3 \mathrm{mM} \mathrm{CaCl}_{2}, 3 \mathrm{mM} \mathrm{KCl}, 10 \mathrm{mM} \mathrm{N}\right.$-tris[hydroxymethyl]methyl2aminoethanesulfonic acid buffer and $25 \mathrm{mM}$ sucrose at $\mathrm{pH}$ 6.9). Treatment groups were injected with the same saline, this time containing either $100 \mu \mathrm{M}$ Methiothepin maleate (TOCRIS, UK) or 10-4 mM 5-HT (Sigma-Aldridge, St. Louis, MO) (Kloppenburg \& Mercer 2008).

Experiment 1: Effects of 5-HT and a 5-HT Antagonist on Detection Thresholds:

The goal of this experiment was to determine the effects of 5-HT and a 5-HT antagonist on the animal's ability to detect odors (CR) across a dilution series. Based on prior research indicating that 5-HT causes spike broadening, we expected that 5HT injection into the ALs would lower detection thresholds with the antagonist having the inverse effect. The experimental design and protocol is depicted in Figure 3. A total of 243 animals were used for this experiment. Animals were conditioned using 2-Hexanone (CS). Twenty-four hours post conditioning, the 
caudal end of the head capsule was opened and the cibarial pump muscle was repositioned forward and secured as described above. Animals were randomly assigned to control $(n=122)$ and drug groups $(n=121)$; the drug group was split in half between $5-\mathrm{HT}$ and methiothepin ( $\mathrm{n}=61$ and $\mathrm{n}=60)$ respectively. Drug and control data was collected in tandem to reduce seasonal effects as well as day by day swings in weather, which can affect moth performance on behavioral tasks. Moths were either injected with saline, 5-HT, or methiothepin. Two researchers performed the test phase; one performed the injections and the other collected the test data and was blind to the contents of the injection. Fifteen minutes post injection, animals were tested across a dilution series of 2-hexanone $(0.05 \mathrm{ng} / \mu \mathrm{L}, 5 \mathrm{ng} / \mu \mathrm{L}$, $50 \mathrm{ng} / \mu \mathrm{L}, 0.5 \mu \mathrm{g} / \mu \mathrm{L}$ and $5 \mu \mathrm{g} / \mu \mathrm{L}$; Daly et al. 2007). As a post-test measure, animals were given $1 \mu \mathrm{L}$ of sucrose to ensure that there were no effects of the surgery/injection on the animal's ability to perform the behavior. 
A.
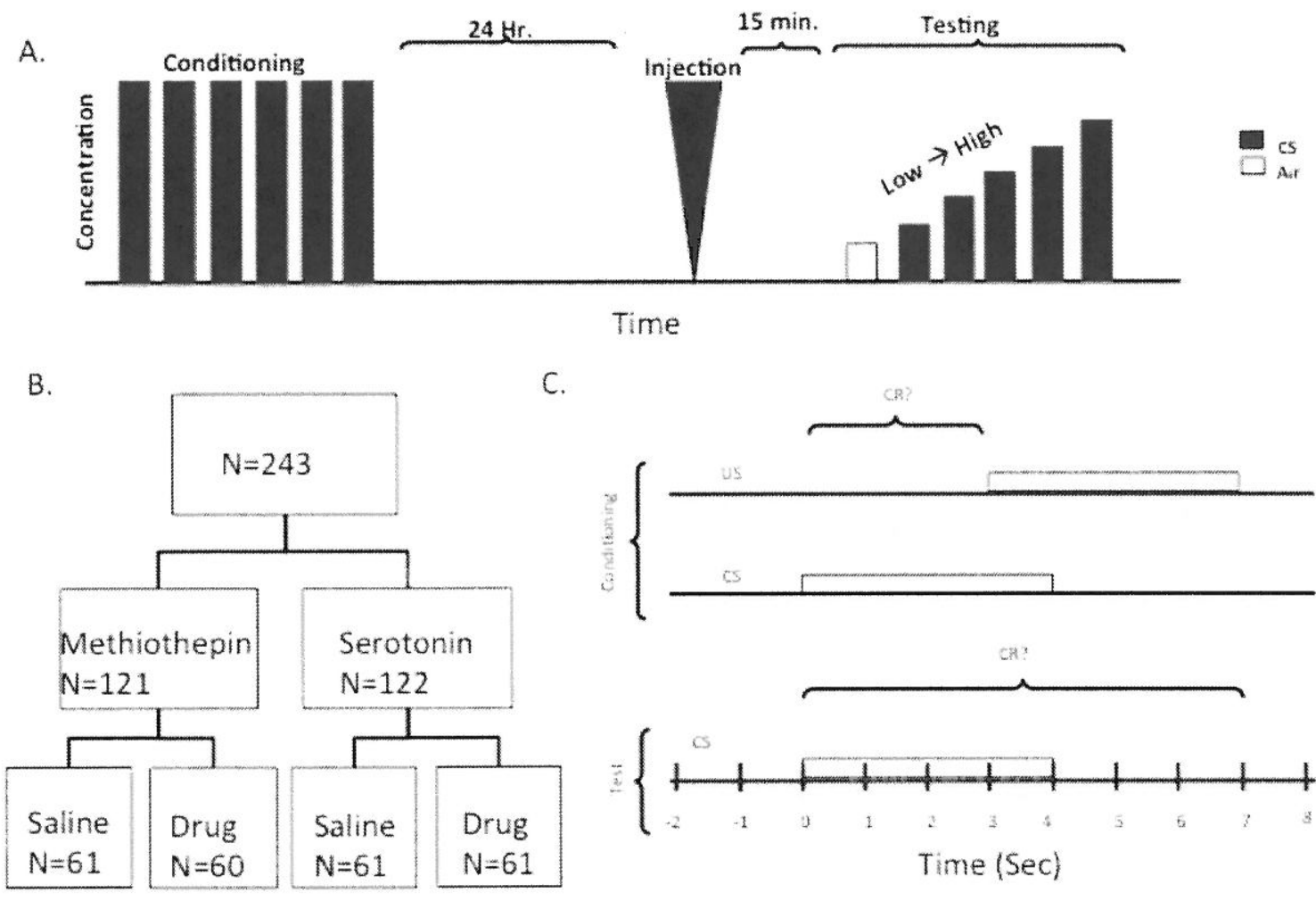

C.
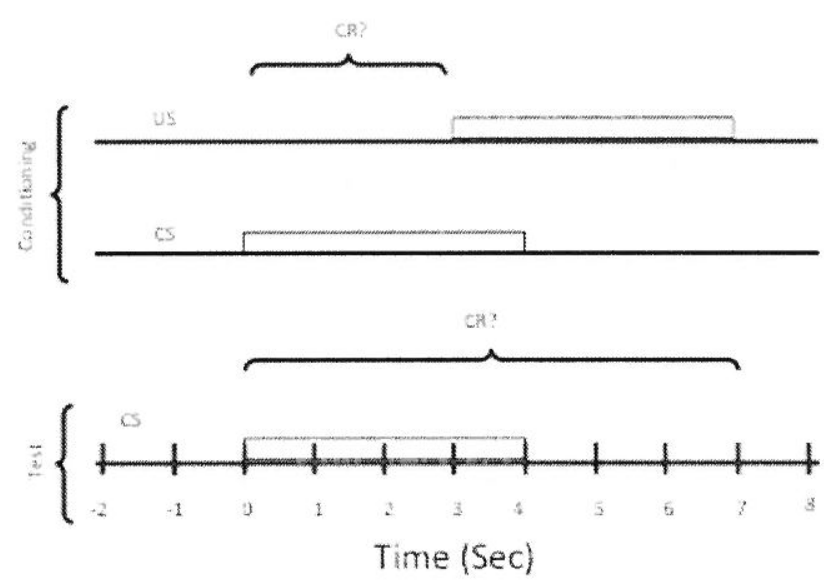

Figure 3: Experimental protocol and design for Experiment 1: Detection Threshold. (A) Panel A depicts the experimental protocol for the detection threshold experiment. 6 conditioning trials were presented to the animal. Twentyfour hours post conditioning, the animal's head was opened and injected with either saline, 5-HT, or methiothepin. Following a 15-minute wait period post injection, the animal was tested across a dilution series from low to high to avoid extinction. (B) Experimental design and numbers of animals assigned to each group for the detection threshold study. Animals were randomly assigned to treatments. (C) Temporal schematic of conditioning paradigm. During conditioning, the conditioned stimulus (CS) was delivered for the first 4 seconds. The onset of the unconditioned stimulus (US) was at 3 seconds with a contiguous 1-second overlap. During testing, a conditioned response (CR) was scored for 7 seconds

Experiment2: Effects of 5-HT and 5-HT Antagonist on Ability to Generalize:

The goal of this experiment was to determine the effects of 5-HT and a 5-HT antagonist on the animal's ability to generalize odors. The experimental design and protocol is depicted in Figure 4. Animals were conditioned as previously described above. 2-Hexanone (CS) was used for conditioning. For the testing phase of this 
experiment, animals were exposed to a series of 3 neat odors; 2-Hexanone (CS), 2Heptanone (S), a chemically similar odor, and Decanol (D), a chemically different odor. Odors were presented in a sequentially from CS, to S, then D.

A total of 241 animals were used for this experiment. Twenty-four hours post conditioning, the animal's head capsule was surgically opened and the ALs were exposed. As before, animals were randomly assigned to control and drug groups (Fig. 4B); injected with saline, 5-HT or methiothepin, using two researchers to inject and test respectively. Animals were again post tested with sucrose as above to confirm ability to feed. 


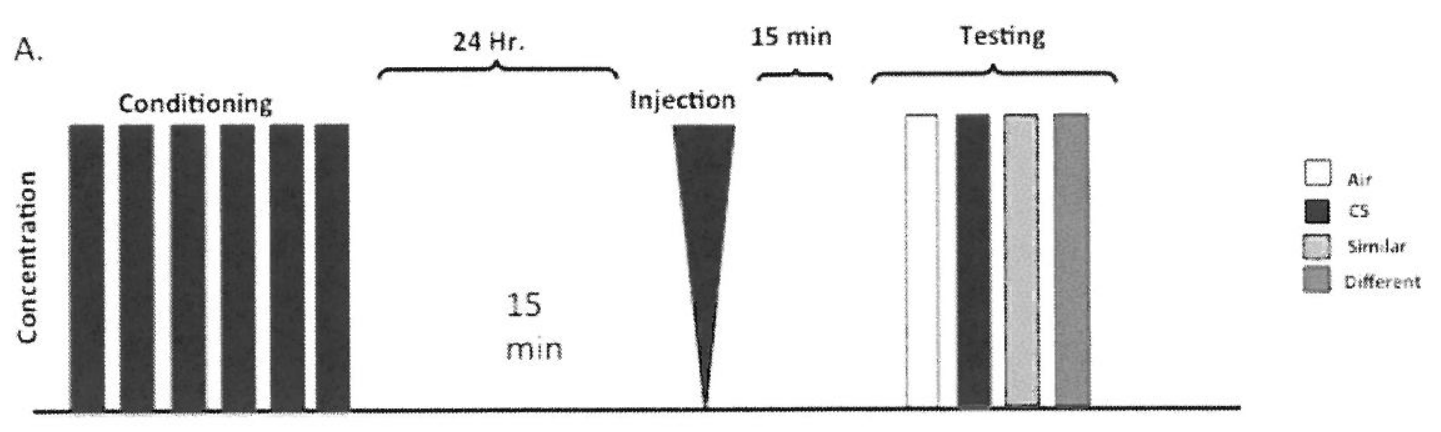

Time

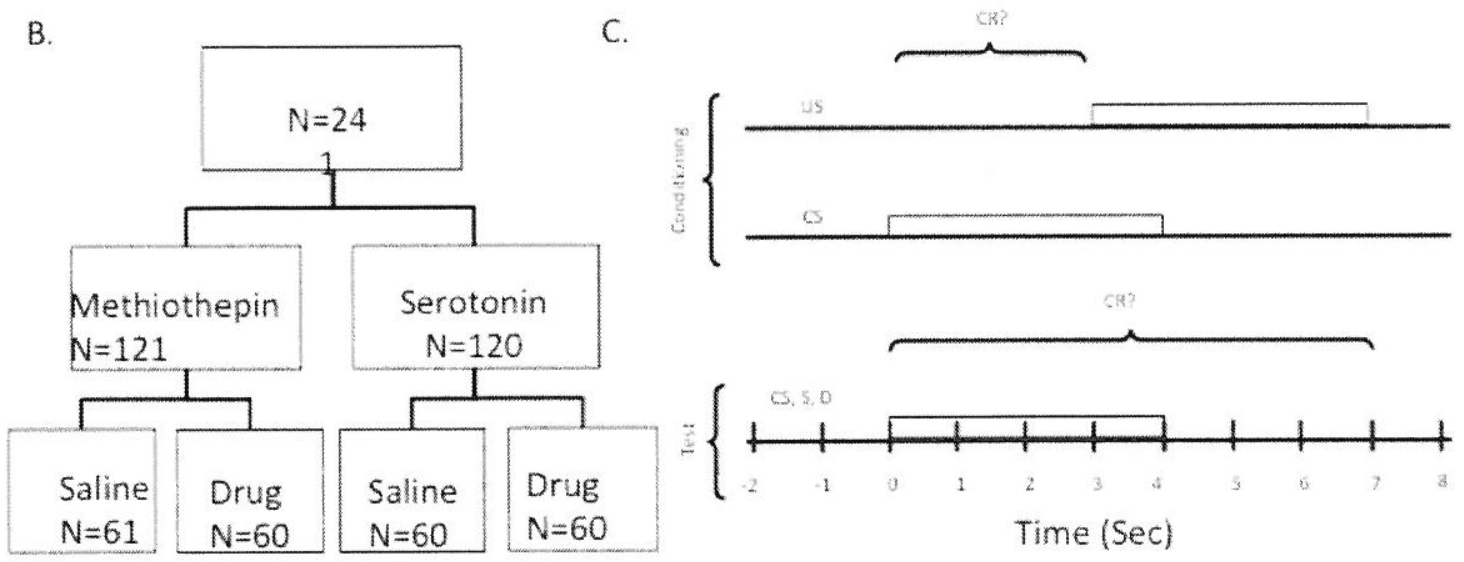

Figure 4: Experimental protocol and design for Experiment 2: CS

generalization. (A) Panel A depicts the experimental protocol for the generalization experiment. 6 conditioning trials were presented to the animal. Twenty-four hours post conditioning, the animals head was opened and injected with either saline, 5HT, or methiothepin. Following a 15 minute wait period post injection, the animal was tested using a blank, 2-hexanone (CS), 2-Heptanone (S), Decanol (D). (B) Experimental design for detection threshold. Distribution of the animals according to treatment. Animals were randomly assigned to treatments. (C) Temporal schematic of conditioning paradigm. During conditioning, the CS was delivered for the first 4 seconds. The onset of the US was at 3 seconds with a contiguous 1-second overlap. For testing, the CR was scored throughout the 7-second presentation.

Analysis:

A general linear model (GLM) was used to quantify differences of the CR in SAS (SAS Institute, North Carolina). In both experiments 1 and 2, there were 5 main effects, sex, age, drug treatment (treat), odor and individual differences (bug); sex, age, and treat were all nested within bug (bug (sex, age, treat)). Sex, age treat and 
odor were all treated as categorical variables. The model seeking to explain CR probability was defined as $\mathrm{CR}=$ sex age treat odor bug(sex age treat) treat*odor sex*treat*odor/ss3. A type 3 sum of squares analysis was used due to the unbalanced number of animals with bug(sex*age*treat) as the error term. Alpha was set at $p=0.05$. A Tukey's Honestly Significant Difference (HSD) post hoc analysis was preformed. In cases were we describe CR probability as a function of either conditioning or odor concentration a second order polynomial was used as it best represented the data based on $\mathrm{R}^{2}$.

For detection thresholds, one-way studentized t-test was conducted across the odor concentrations. Each mean was compared to the air response. The significance level was corrected based on the number of t-test performed. Alpha was set at $p=0.01$ 
Chapter 3: Results 


\section{Results:}

Experiment 1:5-HT Increases Olfactory Responsiveness to 2-Hexanone and 5-HT antagonist has no effect.

The aim of this experiment was to determine the role of 5-HT in olfactory function. The proposed hypothesis was that 5-HT will enhance the ability of the moth to detect odors. Thus, I expect moths will detect odors at lower concentrations when 5 -HT is pharmacologically upregulated. This has been previously described in Manduca using pheromone gland equivalents (PGEs) where it is shown to increase responsiveness to lower concentrations of PGEs (Kloppenburg \& Mercer 2008). Figure 5A displays the acquisition of the conditioned feeding response in moths for Experiment 1 . Here I show that as the number of conditioning trials increase, the response probability increases. At conditioning trial 4, the learning curve asymptotes. After multiple conditioning trials the moths start to reduce the frequency in which they respond to the sucrose solution; this well documented effect is believed to be attributable to satiation. As expected, acquisition rate was nearly identical for both treatment groups thus indicating that both groups learned the task approximately equally.

Figure $5 \mathbf{B}$ shows the effect of 5 -HT injection $\left(10^{-4} \mathrm{mM}, 2 \mathrm{~nL}\right.$ volume $)$ on the detection threshold of 2-Hexanone. When tested across the odor dilution series, the 5-HT group demonstrated a higher responsiveness to all of the odor concentrations with the exception of $1 \mu \mathrm{g} / 2 \mu \mathrm{L}$; this is most likely due to noise. T-test comparisons of responses to the blank and each concentration indicated that the detection threshold for a normal moth without surgery is $0.1 \mu \mathrm{g} / 2 \mu \mathrm{L}(p=0.00103)$, this was 
consistent with published data for the detection threshold of 2-Hexanone (Daly et al 2007). The detection threshold for 5 -HT injected moths was calculated to be $0.01 \mu \mathrm{g} / 2 \mu \mathrm{L}(p=0.0018)$, indicating an overall increase in sensitivity with 5 -HT when compared to control moths and 5-HT injected moths were able to detect odors $1 \log$ step lower concentration than control animals. These results suggest that 5-HT enhances olfactory acuity.

The overall statistical model for 5-HT explaining the variation of a moth eliciting a $\mathrm{CR}$ was significant $(\mathrm{F}=4.45, p<0.001)$. The class variable concentration was found to be significant ( $F=13.23, p<0.0001)$, However, when using bug(sex*age*treatment) as the error term, none of the main effects were found to be significant $\operatorname{sex}(\mathrm{F}=0.92, p=0.3391)$, age $(\mathrm{F}=1.23, p=0.2948)$ and treatment $(\mathrm{F}=0.70$, $p=.4033$ ). Figure 6 shows the Tukey grouping of odor concentrations. The grouping shows that there was no significant difference between the concentrations that were grouped (indicated by the letter of the grouping) but there were significant differences between the non-grouped concentrations.

Figure 5C shows the response probability of moths injected with Methiothepin maleate when compared to control animals. There is a proportional relationship of odor concentration and response probability. As the odor concentration increases, the response probability of the animal also increases. The data was fit with a second order polynomial trendline. Both the methiothepin and saline groups followed the same path. This indicates that there was no antagonistic effect on the animal's ability to detect the CS odor at any given concentration. 
The overall statistical model for Methiothepin explaining the variation of a moth eliciting a CR was significant $(\mathrm{F}=4.26, p<0.001)$. Again, however, when the individual variables were tested using bug(sex*age*treatment) as the error term, none of the main effects were found to be significant $\operatorname{sex}(\mathrm{F}=1.23, p=0.2692)$, age $(\mathrm{F}=0.60, p=0.5496)$ and treatment $(\mathrm{F}=1.17, p=0.2818)$. The Tukey post hoc analysis for concentration found that there were significant differences at the 0.05 level between the concentrations of $10-0.001,10-0,0.1-0.001,0.01-0.001,0-0.001$, $0-0.01,0-0.1,0-1$.

A.

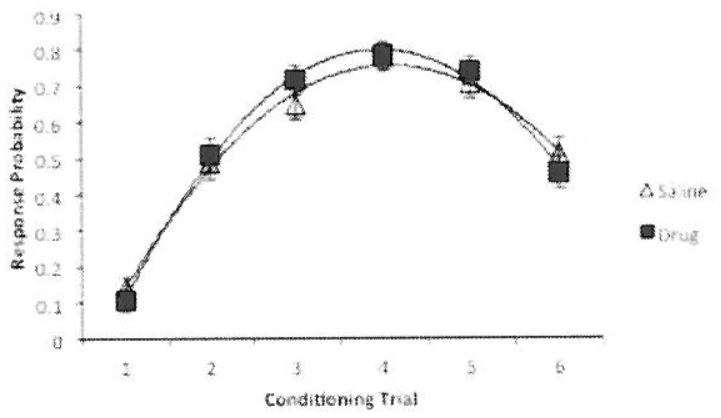

B.

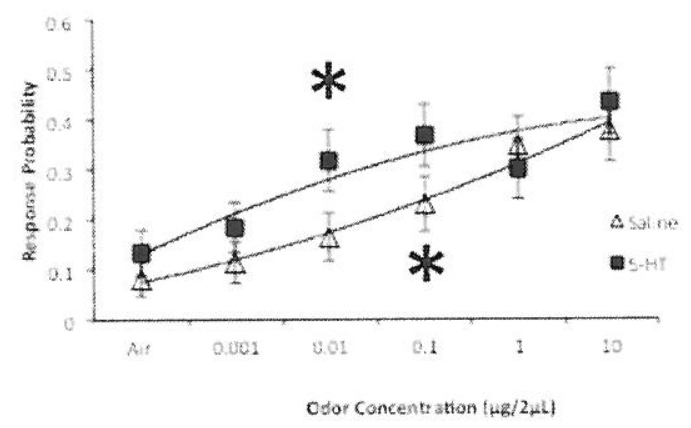

C.

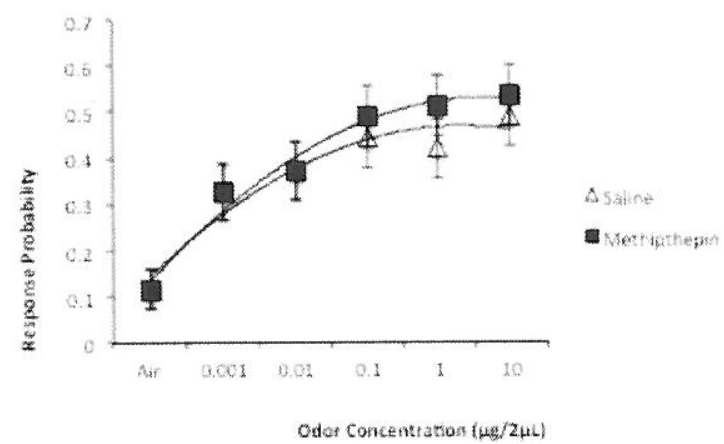

Figure 5: Results of Experiment 1 5-HT Significantly Increases Olfactory Sensitivity: (A) Acquisition Data for Detection Threshold Experiment. This figure depicts the response probability over 6 conditioning trials. The conditioning trials for the saline group have been pooled into one group due to the assumption that all animals learn the same. (B) Detection threshold results for 5-HT. This figure demonstrates the response probability of an animal eliciting a CR. The error bars 
represent standard error of the mean. The data is fitted with a $2^{\text {nd }}$ order polynomial. C) Detection threshold results for Methiothepin. This figure demonstrates the response probability of an animal eliciting a CR. The error bars represent standard error of the mean. The data is fitted with a $2^{\text {nd }}$ order polynomial. The asterisk represents a significant detection threshold.

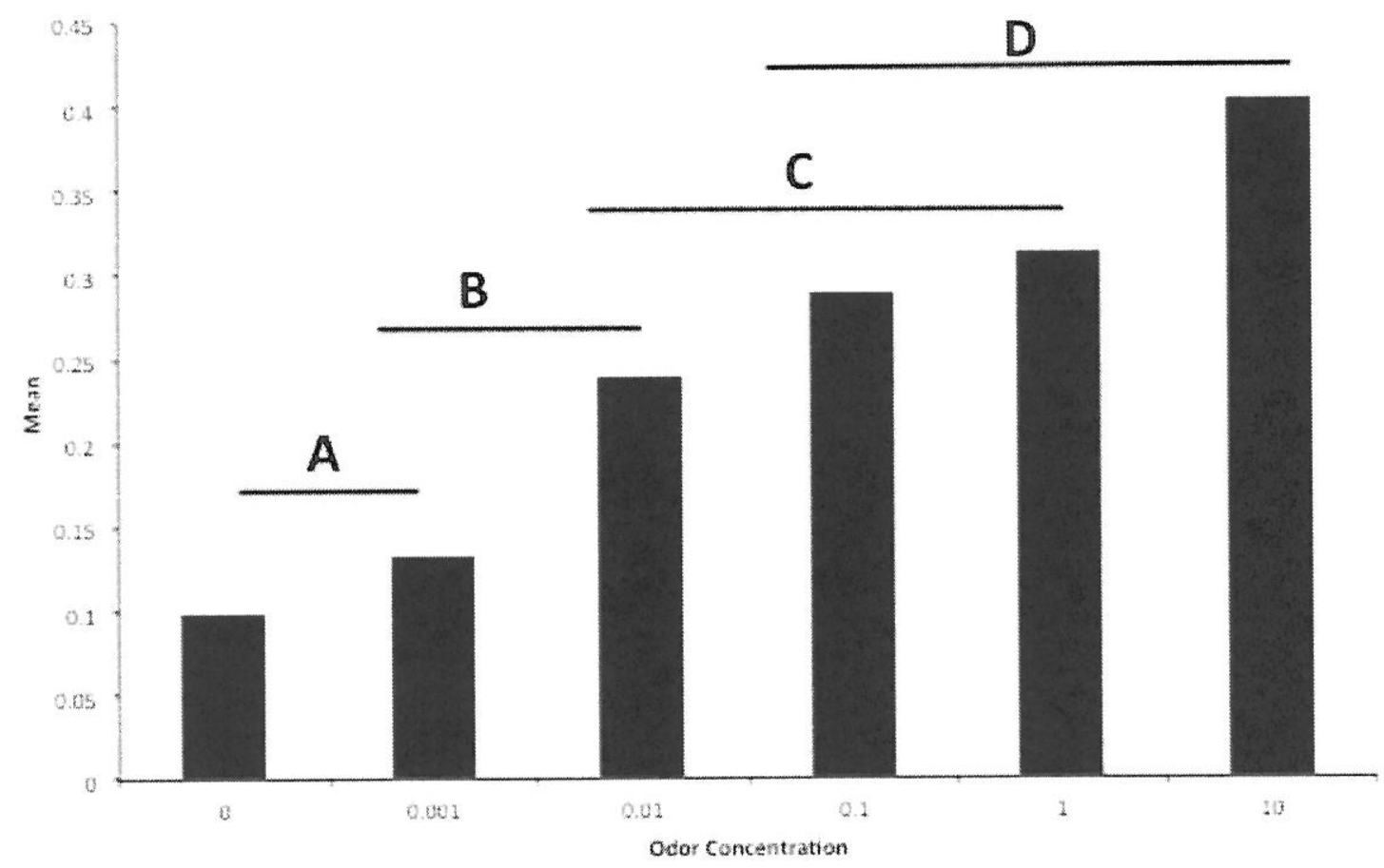

Figure 6 : Tukey's Groups of Odor Concentrations. This figure represents the differences between odor. The concentrations that the bars overlap signify that there is no significant difference between each of the odors.

Experiment 2: 5-HT Increases Olfactory Responsiveness and Sharpens

\section{Generalization Gradients:}

The aim of this experiment was to determine the role of 5-HT function on the moth's ability to discriminate odors as measured behaviorally with a CS generalization protocol. The hypothesis for this experiment was 5-HT will decrease generalization of the conditioned response from the $C S$ to $S$ then D odors. Figure 7A displays the acquisition of the animals. Similar to the detection threshold study, 
animals in both the drug group and the saline group followed the same trend. The CR probability increased as the number of conditioning trials increase until the learning curve asymptotes at conditioning trial 4 where it then declines. This indicated that the animals were learning the task approximately equally independent of the treatment group to which they were assigned to.

Figure 7B displays the results of the generalization experiment. The moths responded with a higher response probability when injected with 5-HT as compared to control. The increase in responsiveness to the stimulus was seen throughout the presentation of all the neat odors and to the blank. This indicated that there was an increase in the overall responsiveness of the animal. If the animals are responding higher to the blank, this suggests that the moth is aroused which is consistent with fluctuating levels of 5-HT during the photophase cycle. Correspondingly, there was an increased responsiveness for both the CS and the S odors. The similarity in responsiveness indicated that moths generalized the $\mathrm{S}$ odor. For the $\mathrm{D}$ odor, animals with injected with 5-HT responded similarly to that of the blank. This indicated that they do not generalize learning about CS to the D odor, which was to be expected due to the extreme difference in the molecular structure of the 1-Decanol. Since the CR probability to the CS and S odors increased but the CR to D did not, this implies that the generalization gradient became steeper; this is confirmed by the inset linear regression functions (Fig. 7B).

Consistent with this interpretation, moths injected with methiothepin where generally less responsive, as compared to controls. There was also a decrease in 
responsiveness to the blank. Again, there was no difference in the overall responsiveness to the $\mathrm{D}$ odor.

The overall statistical model for experiment 2 explaining the variation of a moth eliciting a $\mathrm{CR}$ was significant $(\mathrm{F}=2.76, p<0.001)$. The class variable odor was found to be significant $(\mathrm{F}=28.22, p<0.0001)$. When the specified hypothesis were tested using bug(sex*age*treatment) as the error term, two of the main effects were found not to be significant $\operatorname{sex}(\mathrm{F}=0.84, p=0.3598)$, and age $(\mathrm{F}=1.03, p=0.3578)$. There was however a significant effect of treatment ( $\mathrm{F}=3.39, p=0.0187)$ when the bug(sex*age*treat) was used as the error term. The post hoc analysis for odor found that there were significant differences between the $\mathrm{S}$ and D odor, CS and S relative to the control, and the D odor to all of the odors presented. The post hoc analysis for treatment found that there were significant differences between the drug and the saline groups. There was also a significant difference between the two drug groups. 
A.

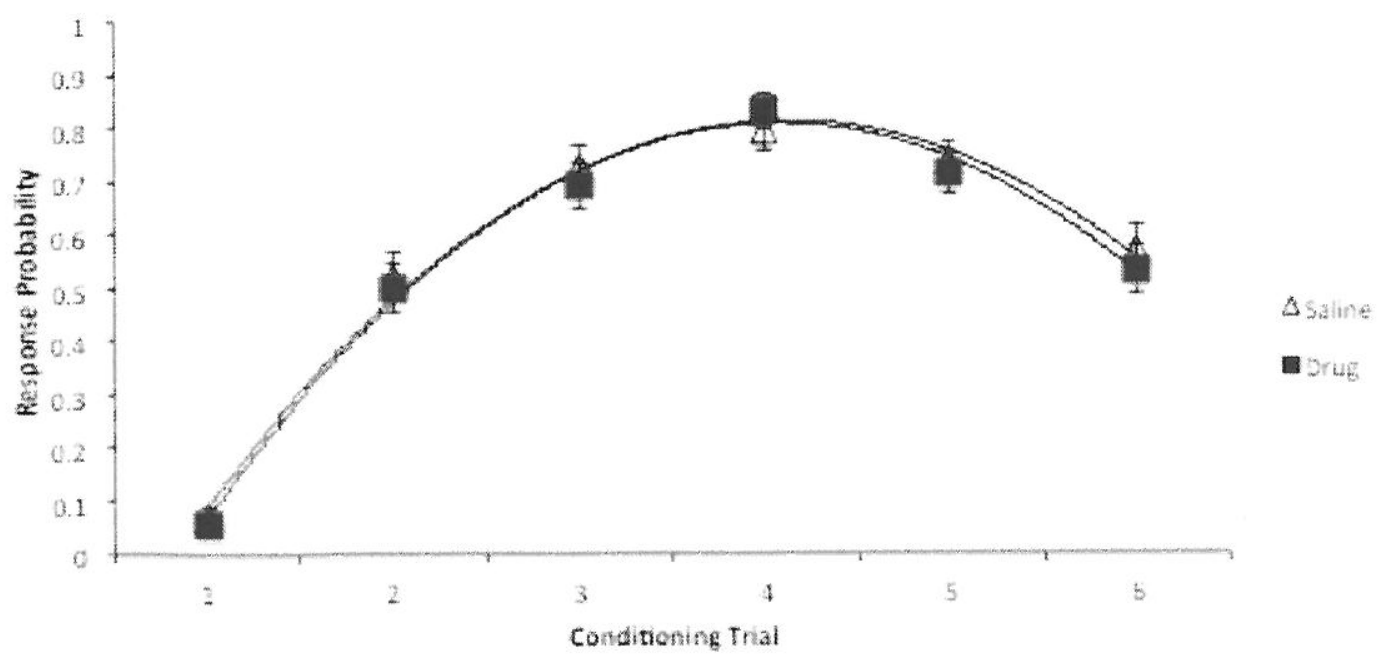

B.

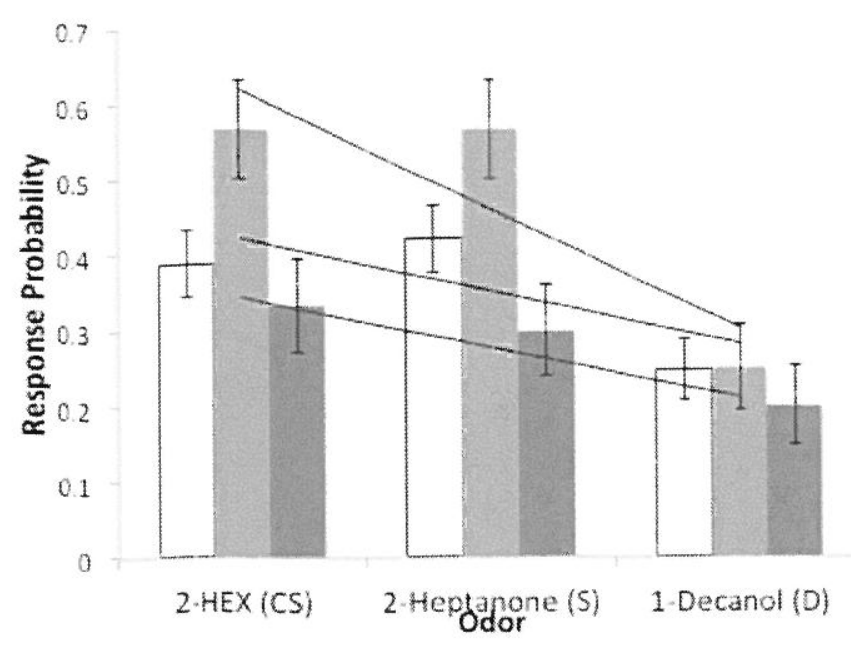

C.

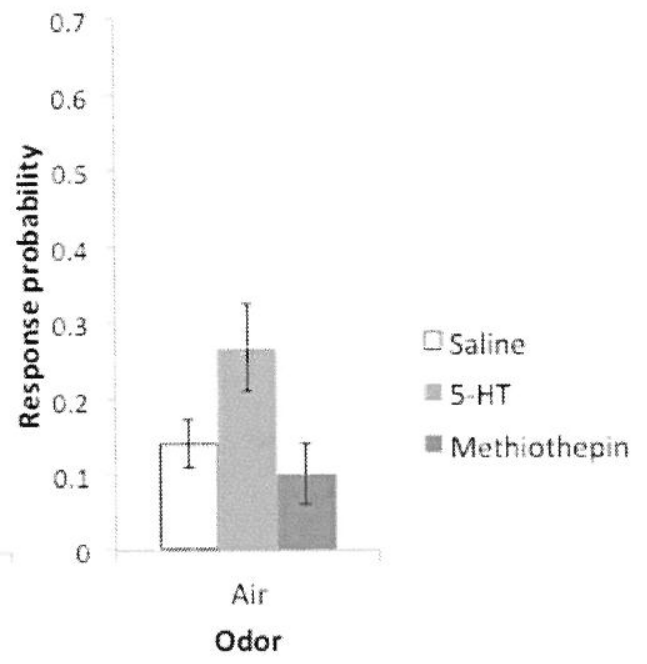

Figure 7: Results of Experiment 2: 5-HT Sharpens the Generalization Gradient: (A) Acquisition Data for Generalization Experiment. This figure depicts the response probability over 6 conditioning trials. The conditioning trials for the saline group have been pooled into one group due to the assumption that all animals learn the same. At conditioning trial 4 the learning curve starts to asymptote due to lack of motivation to feed. (B) This figure depicts the CR probability across odors. The error bars represent standard error of means. The linear trend line indicates the generalization gradient. (C) This panel depicts the response probability of the animal to air by treatment. 
Chapter 4: Discussion and Future Directions 


\section{Discussion:}

Neuromodulators alter the efficacy of a circuit/system based on the physiological state of the animal and in order to accommodate and/or account for changes in contextual demands. The needs of the animal change throughout the day whether they are seeking a mate, sleeping, or foraging. Understanding how neuromodulators work is critical to understanding how circuits in the brain are are tuned to process variable amounts of information that the animal encounters. 5-HT is a known neuromodulator that has been show to affect the overall sensitivity of the olfactory system in Manduca. 5-HT alters the system through changes in channel conductances thus altering the membrane properties of the cell (Kloppenburg et al. 1999, Mercer et al, 1996). Similar effects of 5-HT are seen in the presence of odor-evoked responses where there is still an overall excitability of the cell in response to the odor presentation when 5-HT is applied. (Kloppenburg \& Heinbockel 2000; Dacks et al. 2008, Dacks et al. 2009).

Although there is only a single source of 5-HT into the AL, understanding how 5 -HT is modulating olfactory function is a complex problem. The single souce if 5-HT is a bilateral pair of CSD neurons in the AL along with 4 different 5-HT receptors. (Kent et al. 1987, Dacks et al. 2006b; Dacks et al. 2013). These neurons ramify all of the 63 glomeruli of the AL. In addition to indirectly modulating sensory input, these cells also directly modulate PN output from the AL as two-thirds of the connections between the CSD neuron and the other cells are the output synapses from the 5-HT cell (Kloppenburg \& Mercer 2008). The working hypothesis of this experiment was application of 5-HT will increase olfactory sensitivity resulting in 
increased the conditioned feeding response, there by suggesting that 5-HT up regulates olfactory sensitivity.

A common theme in the previous studies that look at 5-HT modulation in the olfactory system is an increase in the sensitivity of the system leading to an increase in overall responsiveness. These results are consistent with the present study. We observed that when 5-HT was injected into the AL, that there was an increase of overall responsiveness. This indicated that there might be a decrease in the resistance of the cell thus lessening the amount of stimulus needed to evoke an action potential in the cell.

Serotonin significantly increases olfactory sensitivity whereas disrupting 5-HT has no effect on detection

5-HT has been shown to have a behavioral consequence in animals. 5-HT induces an increased olfactory sensitivity and which allows for the detection of pheromones at lower concentrations when compared to control animals (Gatellier et al 2004; Kloppenburg et al. 2008). the current experiment differs from previous behavioral experiments because we observed behavioral consequence to nonpheromone odors. In Gatellier et al. (2004), researchers used an agonist/antagonist approach to understanding the behavioral response to 5-HT. One limitation to their study was the antagonists that they used, Ketanserin and Mianserin, which have a high affinity for octopamine receptors, another known neuromodulator of insect olfactory processing. Thus these agents could have a secondary effects on the olfactory circuit by altering the octopamine pathway. Their results suggest that 
there is a decrease in pheromone detection; however during the wash phase the effect did not wash out. Our study selected an antagonist that has a specific binding affinity for 5-HT1 $1_{B}$ receptors. Another limitation to the Gatellier et al. (2004) experiment was the inability to localize the effect of 5-HT to just pheromone detection. Our study allowed for localized the injection of 5-HT to the AL, which would eliminate confounding effects with other circuits, such as the motor circuit.

In this study, we observed an enhancement of olfactory responsiveness with the injection of 5-HT in the AL. The animals that received 5-HT were able to detect all concentrations, including blanks, with a higher response probability when compared to control moths. In all cases except the $1 \mu \mathrm{g} / 2 \mu \mathrm{L}$ concentration of odor, there was a higher response probability when compared to control animals. The source of this anomaly is unclear. It was hypothesized that an increase in input resistance induced by 5-HT would lead to less input to the ORCs in order to elicit a response in AL neurons thus shifting the threshold for activation (Kloppenburg et al. 1999). This is concurrent with the results of the detection threshold study. In moths that are not surgically injected, the typical detection threshold for 2-hexanone is $0.1 \mu \mathrm{g} / 2 \mu \mathrm{L}$ (Daly et al. 2007).

It was determined that moths had a large increase in responsiveness one log step early when compared to the control group. Because the two groups were randomly collected in tandem, we eliminated any confounding effects such as changes in weather and seasonal changes. Interestingly, an increase response to the blank was observed indicating an overall enhancement in sensitivity in the olfactory circuit. It was not until the $0.01 \mu \mathrm{g} / 2 \mu \mathrm{L}$ that a significant increase in the 
responsiveness of the animal was observed when compared to control animals. In the control animals, we observed an increase in response probability as the odor concentration is increased. This was to be expected since higher concentrations of odors are easier to detect than lower concentrations. Overall, 5-HT is increased the response probability suggesting that there was an increase in sensitivity in the olfactory circuit, typical of neuromodulation. The behavioral consequence that we observed was consistent with the effect of electrically evoked potentials and the odor evoked responses. Changes in the firing properties through the alteration of 2 $\mathrm{K}^{+}$channel conductances lead to an overall increase in the sensitivity of the system.

Along with changing the firing properties of the cell, neuromodulators also alter the physiological properties of the cell. In the Manduca AL, there are four different receptor types, Ms5HT1 $1_{A}, M_{s} 5 H T 1_{B}$, Ms5-HT2, and Ms5-HT7 (Dacks et al. 2006b, Dacks et al. 2013). Each receptor type is coupled to different second messenger systems. 5-HT1 receptors couple to inhibitory G-coupled proteins and inhibit the post-synaptic cell, 5-HT2 link to a PKC pathway and increase $\mathrm{IP}_{3}$ and DAG and are excitatory while 5-HT7 receptors couple to PKA pathway and increase cAMP (Nichols \& Nichols 2008).

5-HT may be activating the cAMP second messenger system. This pathway would increase the amount of cAMP and delay the onset of the $\mathrm{I}_{\mathrm{A}}$ and $\mathrm{I}_{\mathrm{K}(\mathrm{V})}$ delayed rectifying current channel. This, in turn, would increase the amount of intracellular calcium concentraion and cause potentiation of the action potential thus prolonging the amount of time that the neurotransmitter is present in the synaptic cleft. This increase in membrane resistance leads to increased time constants that could 
provide the chance for multiple ORCs to temporally summate and initiate a response (Dacks et al 2008). The increased responsiveness to the blank and much lower concentrations of odor could be attributed to the temporal summation of multiple ORCs thus increasing the responsiveness of the moth to the stimulus.

Methiothepin has been shown to antagonize the Ms5HT1b receptor (Dacks et al. 2013). The 5-HT1 receptor is known to be inhibitory in nature thus decreasing the amount of cAMP when activated. However, methiothepin had no overall effect on decreasing olfactory acuity. This could be attributed to 2 possible explanations: 1) The overall concentration of methiothepin was not sufficient, or 2) Methiothepin is not a potent antagonist in Manduca. The concentration of methiothepin for this study was determined from an in vitro study; while $100 \mu \mathrm{M}$ may have been sufficient for cell culture, the dose may have not been high enough to globally affect the AL function. To be corrected, a dose-response curve experiment should have been preformed to determine the most effective dose. This decrease in the cAMP would lead inhibition of the post-synaptic cell.

Another possible explanation as to why methiothepin did not appear to antagonize the system could be due to limited antagonism. In the antennal lobe, we know that there are 4 different receptors types of which we only antagonized one. The problem is that we do not know where these specific receptor types are expressed in the system. Furthermore, the method that we used may have not sufficiently answered the question that we sought to answer. 


\section{5-HT Sharpens the Generalization Gradient and 5-HT Antagonism has no}

\section{Broadening Effect on Generalization Gradients.}

Similar to the detection threshold study, 5-HT has been shown to enhance CR probability in a generalization assay with the exception of $\mathrm{D}$ (1-decanol) due to its difference in molecular structure of 1-decanol. In previous generalization studies, when moths were trained with 2-hexanone and presented with 1-decanol, the CR was significantly decreased suggesting that the moth did not have the ability to generalize 1-decanol to 2-hexanol (Daly et al 2001). Based on previous research it would be expected that there would be an increase in the CR to molecularly similar odors; however, in this study, the moths elicited the exact same mean CR. This may be due to the odors selected. 2- hexanone and 2-heptanone only differ by 1 carbon unit. Perception of these odors to the moth is non-discriminable and would theoretically produce the same response. This is consistent with findings of discrimination studies where moths were unable to discriminate between hexanone and heptanone; the lack of separation of the CS and S odors was indicative of this (Daly et al. 2001).

When looking at the generalization gradient for $5-\mathrm{HT}$, we see that there is a shift in the slope of the line indicating that there is a sharpening effect of the generalization gradient. When the generalization gradient is sharpened, the similar odor is moved closer to the conditioned stimulus along the gradient, whereas the different odor is moved further away from the CS. The shift in the gradients leads to an enhancement of contrast between the odors, meaning that the moth is more able to generalize the similar odor and less likely to generalize the dissimilar. 
Further studies to improve this experiment would be to randomly present the odors during the testing phase and not presented in the same repertoire each trial as previously described in prior research (Daly et al 2001; Mwilaria et al. 2008). Randomizing the presentation of the test odors can eliminate confounding variables such as extinction of a response, especially in the case of two very similar odors (CS \& S).

In order to further understand the neuromodulatory role of 5- $\mathrm{HT}$ in enhancing olfactory acuity to non-pheromone odors, additional behavioral studies need to be conducted. For example, it would be beneficial to understand the moths' ability to discriminate odors with 5-HT application. The combination of these three studies would give a more complete depiction of olfactory acuity. The antagonistic effect on 5-HT receptors also needs to be further characterized. Currently, there is not an effective 5-HT receptor antagonist specialized for Manduca 5-HT receptors. For example, using 5-hydroxytryptophan (5-HTP), a biochemical precursor to 5-HT, could be applied to agonize the system and alpha methyltryptamine can alter the biosynthesis of 5-HT. However, this approach may be difficult requiring surgery, injection with the drugs, then kept alive and healthy for an extended period of time to allow time of the 5-HTP conversion to 5-HT. Naturally altering the biosynthesis of 5-HT could add in more confounding factors such as changing endogenous level of 5-HT in other regions of the nervous system instead of isolating it to the AL; however, as of now, there is no effective antagonist pharmacological agent for Manduca sexta. 
In addition to altering the biosynthesis of 5-HT, manipulating levels of 5-HT during different phases of the circadian rhythm could give further insight into how 5-HT modulates the nervous system. Levels of 5-HT fluctuate throughout the light/dark cycle in Manduca, 5-HT is higher during scotophase (dark cycle) where the moth is actively seeking food and a mate whereas during photophase (light cycle), the level of endogenous 5-HT is low. In this experiment, we manipulated 5HT during the scotophase when levels are already high. To obtain a more complete picture of how neuromodualtion is occurring, 5 -HT would need to be manipulated during photophase as well. Increasing 5-HT levels during scotophase may enhance olfactory acuity and may alter the circuit after exogenously applying a neuromodulatory substance can alter the circuit significantly (Marder 2012).

In conclusion, this study demonstrated that there is an overall enhancement of olfactory responsiveness with the application of 5-HT directly injected into the AL. Manduca were able to significantly detect lower concentrations of a CS (2Hexanone) and able to enhance the overall behavioral responsiveness in the generalization gradient. This study has given a functional role of 5-HT application in Manduca olfactory behavior. 


\section{References Cited}

Christensen, T.A. \& Hildebrand, J.G. (1987). Male-specific, sex pheromone-selective projection neurons in the antennal lobes of the moth Manduca sexta. Journal of Comparative Physiology A, 160: 553-569

Christensen, T.A. \& Hildebrand, J.G. (2002). Pheromonal and host-odor processing in the insect antennal lobe: How different? Current Opinion in Neurobiology, 12: 393-399.

Dacks, A.M., Christensen, T.A. \& Hildebrand, J.G. (2006a). Phylogeny of a serotoninimmunoreactive neuron in the primary olfactory center of the insect brain. The Journal of Comparative Neurology, 498: 727-746.

Dacks, A.M., Christensen, T.A. \& Hildebrand, J.G. (2008). Modulation of olfacatory information processing in the antennal lobe of Manduca sexta by serotonin. Journal of Neurophysiology, 99: 2077-2085.

Dacks, A.M., Dacks, J.B., Christensen, T.A. \& Nighorn, A. J. (2006b). The cloning of one putative octopamine receptor and two putative serotonin receptors from the tobacco hawkmoth, Manduca Sexta. Insect Biochemistry and Molecular Biology, 36: 741-747.

Dacks, A.M., Green, D.S., Root, C.M., Nighorn, A.J., \& Wang, J.W. (2009). Serotonin modulates olfactory processing in the antennal lobe of Drosophila. Journal Neurogenetics, 23: 366-377.

Dacks, A.M., Reale, V., Pi, Y., Zhang, W., Dacks, J.B., Nighorn, A.J., \& Evans, P.D. (2013). A characterization of the Manduca sexta serotonin receptors in the context of olfactory nueromodulation. PLoS One, 8(7): 1-15.

Daly, K.C., Carrell, L.A. \& Mwilaria, E. (2008). Characterizing psychophysical measures of discrimination thresholds and the effects of concentration on discrimination learning in the moth Manduca Sexta. Chemical Senses, 33:95106.

Daly, K.C., Carrell, L.A. \& Mwilaria, E. (2007). Detection versus perception: physiological and behavioral analysis of olfactory sensitivity in the moth (Manduca Sexta). Behavioral Neuroscience, 121: 794-807.

Daly, K.C., Chandra, S., Durtschi, M.L., \& Smith, B.H. (2001). The generalization of an olfactory-based conditioned response reveals unique but overlapping odour representations in the moth Manduca sexta. The Journal of Experimental Biology, 204: 3085-3095. 
Daly, K.C. \& Smith, B.H. (2000). Associative olfactory learning in the moth Manduca sexta. The Journal of Experimental Biology, 203: 2025-2038.

Gatellier, L., Nagao, T. \& Kanzaki, R. (2004). Serotonin modifies the sensitivity of the male silkworm to pheromone. The Journal of Experimental Biology, 207: 2487-2496.

Hildebrand, J.G. \& Shepherd, G. (1997). Mechanisms of olfactory discrimination: Converging evidence for common principles across phyla. Annual Review of Neuroscience, 20: 595-631.

Hill, E.S., Iwano, M., Gatellier, L. \& Kanzaki, R. (2002). Morphologyand physiology of the serotonin-immunoreactive putative antennal lobe feedback neuron in the male silkmoth Bombyx mori. Chemical Senses, 27: 475-483.

Hill, E.S., Okada, K., \& Kanzaki, R. (2003). Visualization of modulatory effects of serotonin in the silkmoth antennal lobe. The Journal of Experimental Biology, 206: 345-352.

Katz, P. \& Frost, W.N. (1996). Intrinsic neuromodulation: Altering neuronal circuits from within. Trends in Neuroscience, 19: 54-61.

Kent, K.S., Hoskins, S.G. \& Hildebrand, J.G. (1987). A novel serotoninimmunoreactive neuron in the antennal lobe of the sphinx moth Manduca sexta persists throughout postembryonic life. Journal of Neuobiology, 18(5):451-465.

Kloppenburg, P., Ferns, D. \& Mercer, A. (1999). Serotonin enhances central olfactory neuron responses to female sex pheromone in the male sphinx moth Manduca Sexta. The Journal of Neuroscience, 19(19): 8172-8181.

Kloppenburg, P. \& Heinbockel, T. (2000). 5-hydroxytryptamine modulates pheromone-evoked local field potentials in the macroglomerular complex of the sphinx moth Manduca Sexta. The Journal of Experimental Biology, 203: 1701-1709.

Kloppenburg, P. \& Hildebrand, J.G. (1995). Neuromodulation by 5hydroxytryptamine in the antennal lobe of the sphinx moth Manduca Sexta. The Journal of Experimental Biology, 198: 603-611.

Kloppenburg, P. \& Mercer, A. (2008). Serotonin modulation of moth central olfactory neurons. The Annual Review of Entomology, 53: 179-190.

Lee, J.K., Strausfeld, N.J. (1990). Structure, distribution and number of surface sensilla and their receptor cells on their olfactory appendage of the male moth Manduca sexta. Journal of Neurocytology, 19: 519-538. 
Marder, E. (2012). Neuromodulation of neuronal circuits: Back to the future. Neuron, 76:1-11.

Mercer, A., Kloppenburg, P. Hildebrand, J.G. (1996). Serotonin-induced changes in the excitability of cultured antennal lobe neurons of the sphinx moth Manduca sexta. Journal of Comparative Physiology A, 178: 21-31

Mwilaria, E., Ghatak, C. \& Daly, K. (2007). Disruption of $\mathrm{GABA}_{\mathrm{a}}$ in the insect antennal lobe generally increases odor detection and discrimination thresholds. Chemical Senses, 10: 1093-1108.

Nichols, D.E. \& Nichols, C.D. Serotonin receptors. (2008). Chemical Review, 108: 1614-1641.

Recorla, R.A. \& Wagner, A.R. (1972). A theory of Pavlovian conditioning: Variations in the effectiveness of reinforcement and nonreinforcement. In A.H. Black \& W.F. Prokasy (eds.) Classical conditioning II: Current Research and Theory (pp. 64-99). New York: Appleton-Century-Crofts.

Rospars, J.P. \& Hildebrand, J.G. (1992). Anatomical identification of glomeruli in the antennal lobes of the male sphinx moth Manduca sexta. Cell Tissue Res. 270:205-227.

Rospars, J.P. \& Hildebrand, J.G. (2000). Sexually dimorphic and isomorphic glomeruli in the sphinx moth Manduca sexta. Chemical Senses, 25: 119-129.

Sun, X.J., Tolbert, L.P. \& Hildebrand, J.G. (1993). Ramification pattern and ultrastructural characteristics of the serotonin-immunoreactive neuron in the antennal lobe of the moth Manduca sexta: A laser scanning confocal and electron miscropic study. Journal of Comparative Neurology 338:5-16. 